\title{
Magnetic Features at the Surfaces of Nanocrystalline Ribbons
}

\author{
M. MigLIERINI ${ }^{a, b, *}$ AND K. ŠAFÁŘOVÁ ${ }^{b}$ \\ ${ }^{a}$ Department of Nuclear Physics and Technology, Slovak University of Technology, Ilkovičova 3, 81219 Bratislava, \\ Slovakia \\ ${ }^{b}$ Centre for Nanomaterial Research, Palacky University, Šlechtitelů 11, 77146 Olomouc, Czech Republic \\ Magnetic behaviour of Nanoperm $\mathrm{Fe}_{90} \mathrm{Zr}_{7} \mathrm{~B}_{3}$ nanocrystalline alloy is studied by surface sensitive techniques \\ comprising atomic force microscopy and magnetic force microscopy. Correlation of structural arrangement \\ and magnetic order including the orientation of net magnetization is obtained by the help of the Mössbauer \\ spectrometry. As-quenched amorphous alloys show no magnetic features. After annealing, maze magnetic domains \\ have developed. Their shape is governed by surface crystallization. Position of net magnetization out of the \\ ribbon plane by about $26^{\circ}$ was determined by the Mössbauer spectrometry.
}

PACS numbers: 68.37.Rt, 75.60.Ch, 75.70.-i, 71.55.Jv, 76.80.+y

\section{Introduction}

Nanocrystalline alloys obtained from amorphous precursors by controlled annealing exhibit unique soft magnetic properties [1] which determine their practical applications [2]. Original metallic glasses are usually prepared in the form of ribbons by rapid quenching on a rotating wheel. This preparation procedure leads to noticeable differences between the bulk and the surfaces of the ribbons [3].

Conventional magnetic measurements provide integral information over different structural components contained in the nanocrystalline alloys (i.e., nanocrystalline grains, amorphous residual matrix, and interconnecting phase). This contribution aims in characterization of magnetic behaviour of Nanoperm nanocrystalline alloy by surface sensitive analytical techniques. Namely, we demonstrate the use of atomic force microscopy (AFM) and magnetic force microscopy (MFM) for investigation of surface structural and magnetic features, respectively. The obtained results are compared with those from ${ }^{57} \mathrm{Fe}$ Mössbauer spectroscopy.

\section{Experimental}

Precursors of $\mathrm{Fe}_{90} \mathrm{Zr}_{7} \mathrm{~B}_{3}$ Nanoperm metallic glasses were prepared by rapid quenching of melt on rotating wheel in form of ribbons. AFM and MFM images were recorded by NTEGRA Aura system in semicontact mode using tips with nominal radius of $30 \mathrm{~nm}$. The Mössbauer spectra were collected by a standard constant acceleration spectrometer with ${ }^{57} \mathrm{Co} / \mathrm{Rh}$ source in transmission geometry. Surface regions down to the depth of about

* corresponding author; e-mail: marcel.miglierini@stuba.sk
$200 \mathrm{~nm}$ were scanned by detection of conversion electrons, the so-called conversion electron Mössbauer spectrometry (CEMS).

\section{Results and discussion}

As-quenched $\mathrm{Fe}_{90} \mathrm{Zr}_{7} \mathrm{~B}_{3}$ alloy is paramagnetic at room temperature and X-ray diffraction (XRD) amorphous. AFM images in Fig. 1a and c show presence of homogeneously distributed structural features upon the side of the ribbon which was exposed to the surrounding atmosphere during quenching, the so-called air side. Corresponding MFM pictures in Fig. $1 \mathrm{~b}$ and d reflect these surface imperfections rather than orientation of magnetic moments which are missing in paramagnetic state.

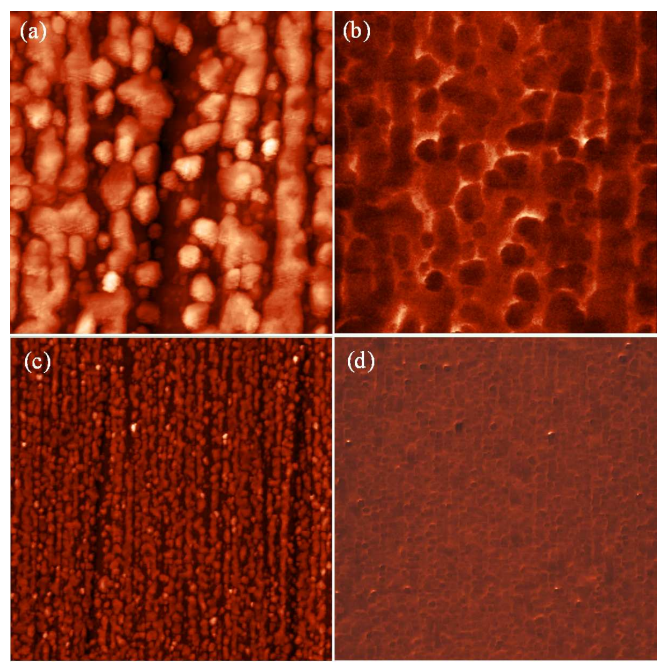

Fig. 1. AFM (a, c) and MFM (b, d) images of as-quenched $\mathrm{Fe}_{90} \mathrm{Zr}_{7} \mathrm{~B}_{3}$ alloy. Air side, $5 \times 5 \mu \mathrm{m}^{2}$ (a), (b), $20 \times 20 \mu \mathrm{m}^{2}(\mathrm{c}),(\mathrm{d})$. 


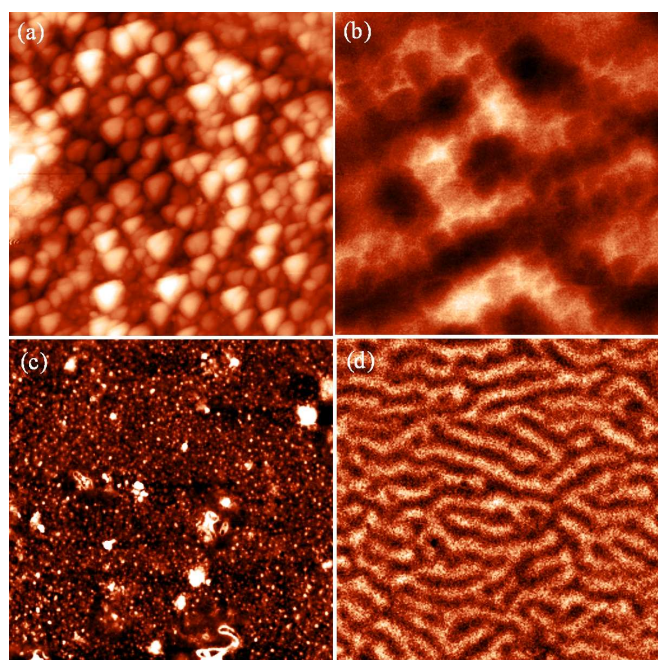

Fig. 2. AFM (a, c) and MFM (b, d) images of $\mathrm{Fe}_{90} \mathrm{Zr}_{7} \mathrm{~B}_{3}$ alloy annealed at $620^{\circ} \mathrm{C} / 80 \mathrm{~min}$. Air side, $5 \times 5 \mu \mathrm{m}^{2}$ (a), (b), $20 \times 20 \mu \mathrm{m}^{2}$ (c), (d).

After heat treatment at $620^{\circ} \mathrm{C} / 80 \mathrm{~min}$ in vacuum, an optimal nanocrystalline structure has evolved. The nanocrystallites form agglomerates that are rather uniform as far as their size is concerned as demonstrated by AFM images in Fig. 2a and c acquired from different air side surface areas. Appearance of ferromagnetic bcc-Fe nanograins affects also magnetic order in the bulk as well as on the surface of the annealed alloy. Subsequently, well developed fine magnetic domain structure is seen in Fig. 2b and d. Dark and bright contrasts correspond to the attractive and repulsive interactions, respectively, between the tip and the sample surface.

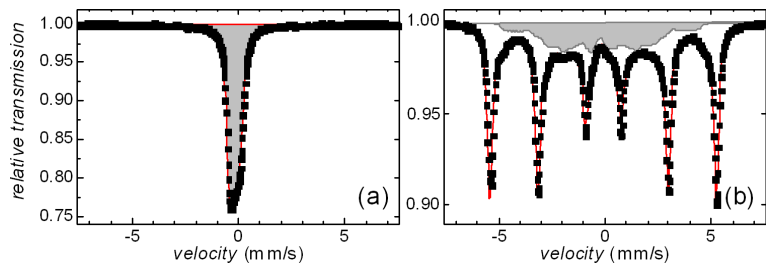

Fig. 3. ${ }^{57} \mathrm{Fe}$ Mössbauer spectra of $\mathrm{Fe}_{90} \mathrm{Zr}_{7} \mathrm{~B}_{3}$ alloy in as-quenched state (a) and after annealing at $620^{\circ} \mathrm{C} / 80 \mathrm{~min}$ (b). Gray spectral components represent the amorphous phase.

The Mössbauer spectrum of an as-quenched sample in Fig. 3a shows broad doublet characteristic of amorphous and paramagnetic alloy. After annealing, bcc-Fe nanocrystals have developed. They are identified by narrow sextet in Fig. 3b superimposed upon broadened (shaded) feature assigned to residual amorphous phase. Sextet-like shape of the latter implies that magnetic interactions have evolved in the originally non-magnetic amorphous alloy. They resulted from extensive exchange interactions among ferromagnetic nanograins

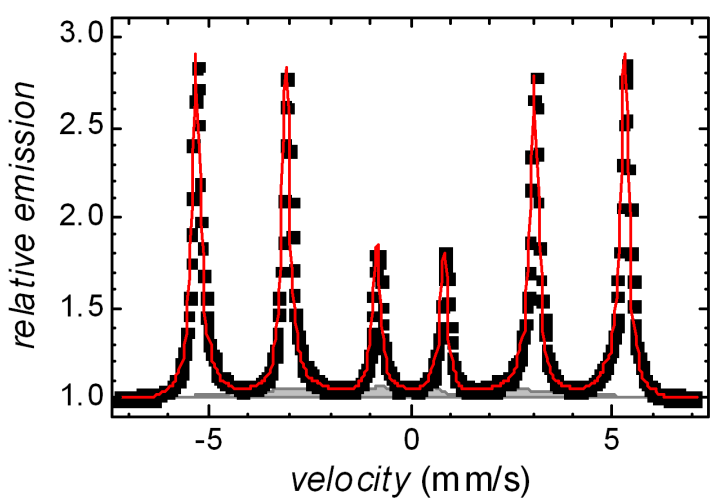

Fig. 4. ${ }^{57}$ Fe CEMS spectrum taken from the air side of the annealed $\left(620^{\circ} \mathrm{C} / 80 \mathrm{~min}\right) \mathrm{Fe}_{90} \mathrm{Zr}_{7} \mathrm{~B}_{3}$ alloy. The grey component represents the amorphous phase.

and changes in chemical short range order of the residual amorphous phase.

Transmission Mössbauer spectra in Fig. 3 provide information about the bulk, i.e. the whole volume of the alloys. On the other hand, CEMS spectrum in Fig. 4 recorded from the air side of the annealed ribbon reflects surface properties. Here, contribution from the amorphous residual matrix is notably smaller due to more pronounced crystallization at the surface (95\%) than in the bulk $(70 \%)$. Consequently, also magnetic domain structure was formed as seen in Fig. 2d. More detailed image in Fig. 2b shows that individual agglomerates have different orientations of magnetic moments. Formation of the so-called finger-print or maze domains is observed. Position of the net magnetization in the annealed sample can be derived from line intensity ratio of the corresponding Mössbauer spectra for bulk (Fig. 3b) and surface (Fig. 4) regions. It is rotated by about $27^{\circ}$ and $26^{\circ}$ out of the ribbon plane, respectively.

Similar maze domain pattern was observed in polished $\mathrm{Fe}_{80.5} \mathrm{Nb}_{6.9} \mathrm{~B}_{12.6}$ as-quenched ribbons [4]. It is explained by internal stresses originating from the quenching process with close relation to the magnetostriction constant [5]. In our case, the domains were formed as a consequence of pronounced crystallization which also introduces compressive stresses. Curved domains separated by $180^{\circ}$ walls were observed in annealed $\mathrm{Fe}_{91} \mathrm{Zr}_{7} \mathrm{~B}_{2}$ alloy which was electrochemically polished [5]. It is noteworthy that our samples were not polished or otherwise treated.

The appearance of the observed maze domains is related to the overcoming of demagnetizing effects in these regions by the magnetoelastic energy. The latter arises from internal stresses introduced during crystallization.

\section{Conclusions}

In conclusion, as-quenched alloy which is paramagnetic shows no magnetic domains. After annealing bcc-Fe crystallites have developed. They are identified in the bulk 
of the samples by transmission Mössbauer spectrometry and on the surface regions by CEMS. AFM and MFM images have proved that the formation of maze magnetic domains is governed by crystallization. From the Mössbauer spectral line intensities, the position of net magnetization is determined to be deflected from the sample plane by about $26^{\circ}$.

\section{Acknowledgments}

This work was supported by the grants MSM6198959218, 1 M6198959201, SK-PL-0013-09, and VEGA 1/0033/10.

\section{References}

[1] K. Suzuki, A. Makino, A. Inoue, T. Masumoto, J. Appl. Phys. 70, 6232 (1991).

[2] G. Herzer, Phys. Scr. T 49, 307 (1993).

[3] M. Pavúk, M. Miglierini, M. Vůjtek, M. Mašláň, R. Zbořil, Y. Jirásková, J. Phys., Condens. Matter 19, 216219 (2007).

[4] O. Životský, K. Postava, L. Kraus, K. Hrabovská, A. Hendrych, J. Pištora, J. Magn. Magn. Mater. 322, 1523 (2010).

[5] K. Suzuki, D. Wexler, J.M. Cadogan, V. Sahajwalla, A. Inoue, T. Masumoto, Mater. Sci. Eng. A 226-228, 586 (1997). 